\title{
基于光谱指数的植物叶片叶绿素含量的估算模型
}

\author{
宫兆宁 ${ }^{1,2,3,4}$, 赵雅莉 ${ }^{1,2,3,4}$, * 赵文吉 ${ }^{1,2,3,4}$, 林川 ${ }^{1,2,3,4}$, 崔天翔 ${ }^{1,2,3,4}$ \\ (1. 首都师范大学资源环境与旅游学院, 北京 $100048 ; 2$. 三维信息获取与应用教育部重点实验室,北京 100048 ; \\ 3. 资源环境与地理信息系统北京市重点实验室,北京 100048 ; \\ 4. 北京市城市环境过程与数字模拟国家重点实验室培育基地, 北京 100048)
}

摘要: 叶绿素是光合作用能力和植被发育阶段的指示器, 是监测湿地植被生长健康状况的重要指标之一; 高光谱遥感技术可以 为植物叶绿素含量的定量化诊断提供简便有效、非破坏性的数据采集和处理方法。为保证被探测叶片面积相同,消除背景反 射、叶片表面弯曲造成的光谱波动及叶片内部变异造成的影响, 研究采用 Field Spec 3 光谱仪加载手持叶夹式叶片光谱探测器, 测定野鸭湖湿地典型植物的叶片高光谱反射率数据, 同时通过分光光度计室内测定相应叶片的叶绿素含量。采用相关性及单 变量线性拟合分析技术, 建立二者的关系模型,包括叶绿素含量与 “三边” 参数的相关模型以及比值光谱指数 (SR) 模型和归一 化差值光谱指数 (ND) 模型, 并采用交叉检验中的 3K-CV 方法对估算模型进行模型精度检验。结果表明: 植物叶片叶绿素含量 与 “三边” 参数大多都呈极显著相关, 相关系数最大达到 0.867 ; 计算光谱反射率组成的比值 (SR) 和归一化 (ND) 光谱指数与叶 绿素含量的决定系数, 总体相关性比较高, 较好的波段组合均为 550-700nm 与 700-1400nm 以及 550-700nm 与 $1600-$ $1900 \mathrm{~nm}$, 与叶绿素含量相关性最好的指数分别是 $\operatorname{SR}(565 \mathrm{~nm}, 740 \mathrm{~nm})$ 和 $\mathrm{ND}(565 \mathrm{~nm}, 735 \mathrm{~nm})$ 。并通过选取相关性最佳的光谱特 征参数, 分别基于 “三边” 参数和 ND 模型指数构建了植物叶片叶绿素含量的估算模型。其中, 基于红边位置(WP_r) 光谱特征 参数和 $\mathrm{ND}(565 \mathrm{~nm}, 735 \mathrm{~nm})$ 光谱指数建立的叶绿素含量估算模型, 取得了较好的测试效果, 检验拟合方程的决定系数 $\left(R^{2}\right)$ 都达 到 0.8 以上,估算模型分别为 $y=0.113 x-78.74, y=5.5762 x+4.4828$ 。通过 $3 \mathrm{~K}-\mathrm{CV}$ 方法进行测试和检验, 植物叶绿素含量估算模 型均取得了较为理想的预测精度, 预测精度的分别为 $93.9 \%$ 及 $90.7 \%$ 。高光谱遥感技术对植被进行微弱光谱差异的定量分析, 在植被遥感研究与应用中表现出强大优势,为植物叶绿素含量诊断中的实际应用提供了重要的理论依据和技术支持。

关键词: 叶绿素含量; 高光谱模型; “三边”参数;光谱指数; 北京野鸭湖湿地植物

\section{Estimation model for plant leaf chlorophyll content based on the spectral index content}

\footnotetext{
GONG Zhaoning ${ }^{1,2,3,4}$, ZHAO Yali ${ }^{1,2,3,4, *}$, ZHAO Wenji ${ }^{1,2,3,4}$, LIN Chuan ${ }^{1,2,3,4}$, CUI Tianxiang ${ }^{1,2,3,4}$

1 College of Resource Environment and Tourism, Capital Normal University, Beijing 100048, China

2 Key Laboratory of 3D Information Acquisition and Application of Ministry of Education, Beijing 100048 , China

3 Key Laboratory of Resources Environment and GIS of Beijing Municipal, Beijing 100048, China

4 Base of the State Laboratory of Urban Environmental Processes and Digital Modeling, Beijing 100048, China
}

\begin{abstract}
Chlorophyll can be an indicator in photosynthesis capacity and vegetation developmental stages, which is also one of important indicators to monitor health status of wetland vegetation growth. Hyperspectral remote sensing technology can provide a simple, effective and non-destructive data acquisition, which can offer processing method for quantifying diagnosis plant chlorophyll content as well. This study used the Fieldspec 3 spectrometer and a plant probe leaf clip spectral detector

基金项目:863 计划课题(2012AA12A308); 国家青年科学基金项目(41101404); 国家基础测绘项目(2011A2001); 北京市教委科技计划面上项 目（KM201110028013）；国土资源部重点实验室开放基金(KLGSIT2013-04); 国家国际科技合作专项资助(2014DFA21620)

收稿日期: 2013-01-25; 网络出版日期: 2014-03-11

* 通讯作者 Corresponding author.E-mail: 880215zyl@163.com
\end{abstract}


to guarantee the spectrum are detected in the same area of the leaf, it is also eliminating the background reflectance, spectral fluctuations caused by bending of the blade surface and the impact caused by leaf internal variability. This study determined the typical wetland plants leaf hyperspectral reflectance data at Wild Duck Lake, and at the same time the corresponding leaf chlorophyll content was measured using a spectrophotometer indoor. The relationship between chlorophyll content and the Trilateral parameters, as well as the ratio of spectral index model (SR) and normalized difference spectral index (ND) were established respectively using linear regression model., then 3-Fold Cross Validation(3K-CV) was used to test the accuracy of the estimation model. The results showed that most of the "trilateral" parameters were significantly correlated with plant leaf chlorophyll content; the maximum correlation coefficient reached 0.867 . The correlation coefficient between ratio (SR) and normalized (ND) and chlorophyll content were high in general. Suitable band combinations were $550-700 \mathrm{~nm}, 700-1400 \mathrm{~nm}, 550-700 \mathrm{~nm}$ and $1600-1900 \mathrm{~nm}$. The best indices with highest correlation with chlorophyll content were SR (calculated from bands $565 \mathrm{~nm}$ and $740 \mathrm{~nm}$ ) and ND (calculated from bands $565 \mathrm{~nm}$ and $735 \mathrm{~nm}$ ). And then by choosing the best correlation spectrum characteristic parameters based on the Trilateral parameters and ND model index, a plant chlorophyll estimation model was constructed. Among them, a chlorophyll content estimation model established by Red edge position (WP_r) of spectral characteristic parameters and ND ( $565 \mathrm{~nm}, 735 \mathrm{~nm})$ spectral index achieved better test results, and $R^{2}$ both reached above 0.8 , the estimation model were $y=0.113 x-78.74, y=5.5762 x+$ 4. 4828. Using $3 \mathrm{~K}-\mathrm{CV}$ method for testing and validation, the prediction accuracies of both plant leaf chlorophyll content estimation models were $93.9 \%$ and $90.7 \%$, respectively. The quantitative analysis of hyperspectral remote sensing technology shows a strong advantage in detecting vegetation weak spectral differences and provides an important theoretical basis and technical support for the practical application in the diagnosis of plant chlorophyll content.

Key Words : chlorophyll content; hyperspectral model; trilateral parameters; spectral index; the plants of Wild Luck Lake wetland in Beijing

湿地植被是湿地生态系统的重要组成成分, 在 维持生态系统结构和功能方面起到十分重要的作 用, 因此成为湿地生态系统监测的重点 ${ }^{[1]}$ 。选择不 同时空尺度上的典型湿地植被的生化参数进行系统 性研究, 不仅可以及时掌握湿地生态系统的动态变 化, 为湿地生态系统价值评价提供重要参数 ${ }^{[2]}$, 同时 还可作为湿地生态系统基本生态价位的划分标准, 并为湿地生态系统的管理、恢复以及重建提供科学 依据。

叶绿素含量与植被的光合能力、发育阶段具有 较好的相关性, 它通常是光合作用能力和植被发育 阶段 (特别是衰老阶段) 的指示器 ${ }^{[3]}$, 因此叶绿素含 量是作为监测湿地植被生长健康状况的重要标志之 一。近些年发展起来的高光谱遥感技术, 由于其具 有波段多且窄的特点, 能直接对植被进行微弱光谱 差异的定量分析, 在植被遥感研究与应用中表现出 强大优势, 同时也为植被生理参数的定量化诊断提 供了简便、快速、有效、非破坏性的数据采集和处理 方法 ${ }^{[4-5]}$ 。植被叶片反射光谱在可见光范围受植被
叶绿素的强烈吸收影响, 反射率较低; 在近红外区域 则受叶片内部结构等影响, 呈现高反射特征 ${ }^{[6]}$ 。国 内外学者开展了许多基于作物叶片反射特征进行叶 绿素浓度或含量预测研究 ${ }^{[7-21]}$ 。许多研究表明, 植 被光谱“红边” 对植被生长状况反映敏感。其定义为 植物反射光谱曲线中斜率最大的点, 位于波长 $690-$ $720 \mathrm{~nm}$ 之间 ${ }^{[10]}$, 实验室实验已经证实了红边拐点的 波长位置和叶片样品的叶绿素浓度具有正相关 ${ }^{[11]}$, Curran 等人发现红边拐点位置与沼泽松枝条的叶绿 素浓度有正相关 ${ }^{[12]}$ 。杜华强等发现: 红边位置、红 边平均反射率、红边位置附近平均反射率、红边斜 率、红边面积、红谷吸收深度、绿峰反射高度、红边归 一化植被指数、红边植被胁迫指数等 9 个马尾松反 射光谱特征参数均与叶绿素含量间存在指数函数 关系 ${ }^{[13]}$

目前应用高光谱遥感技术在监测和诊断植物叶 绿素含量方面已有一些成效, 但是研究方法单一、不 够全面,并且在湿地植物叶绿素含量估算中的应用 研究很少见。因此, 本文主要以北京野鸭湖湿地自 
然保护区内的湿地植物为研究对象。利用室内光谱 仪以加载叶片夹持器测得的植物叶片高光谱反射率 数据及同步测定的叶绿素含量,通过已有的基于高 光谱反射率数据的 “三边” 参数以及初步构造的差值 和归一化光谱指数模型, 分别分析其与叶绿素含量 的相关性, 比较叶绿素含量的估算效果, 以期确立野 鸭湖湿地植被叶绿素含量的监测模型, 进而在认识 湿地植被的恢复速率和生产力状况方面做一些 探索。

\section{1 材料与方法}

\section{1 研究区概况}

野鸭湖湿地自然保护区是北京唯一的湿地鸟类 自然保护区,也是北京最大的湿地自然保护区。位 于北京市延庆县西北部的延庆镇、康庄镇、张山营镇 和延庆农场交界处, 地理位置处于东经 $115^{\circ} 46^{\prime} 16^{\prime \prime}-$ $115^{\circ} 59^{\prime} 48^{\prime \prime}$, 北纬 $40^{\circ} 22^{\prime} 04^{\prime \prime}-40^{\circ} 30^{\prime} 31^{\prime \prime}$ 之间, 北、东、
南三面环山,西南临官厅水库, 是官厅水库延庆辖区 及环湖海拔 $479 \mathrm{~m}$ 以下淹没区及滩涂组成的次生湿 地, 保护区总面积为 $6873 \mathrm{hm}^{2}$, 湿地面积达 $3939 \mathrm{hm}^{2}$ （图 1)。保护区地处延怀盆地之延庆部分, 属于北 京北山隆起区块中的延庆昌平活动断裂区, 是北京 唯一的山间盆地, 地势平坦, 四周高, 中间低, 海拔高 度 480-800m, 协水河自东北向西南贯穿。该区域 属北温带大陆性季风气候, 四季变化较为明显, 气候 凉爽,昼夜温差较大,降水主要集中在夏季的 6-8 月份, 其中降雨高峰期为 7 月下旬至 8 月上旬 ${ }^{[22]}$ 。 区域内湿地植被资源丰富,共有湿地植物 71 科 213 属 357 种 (高等植物)。同时, 丰富的湿地植被资源 也为水禽和各类鸟类提供了优越的受食、隐藏及繁 殖场所, 是候鸟南北迁徙的主要信道, 也是珍稀水禽 的重要停歇地, 具有极其重要的生物多样性保护 价值 ${ }^{[23]}$ 。

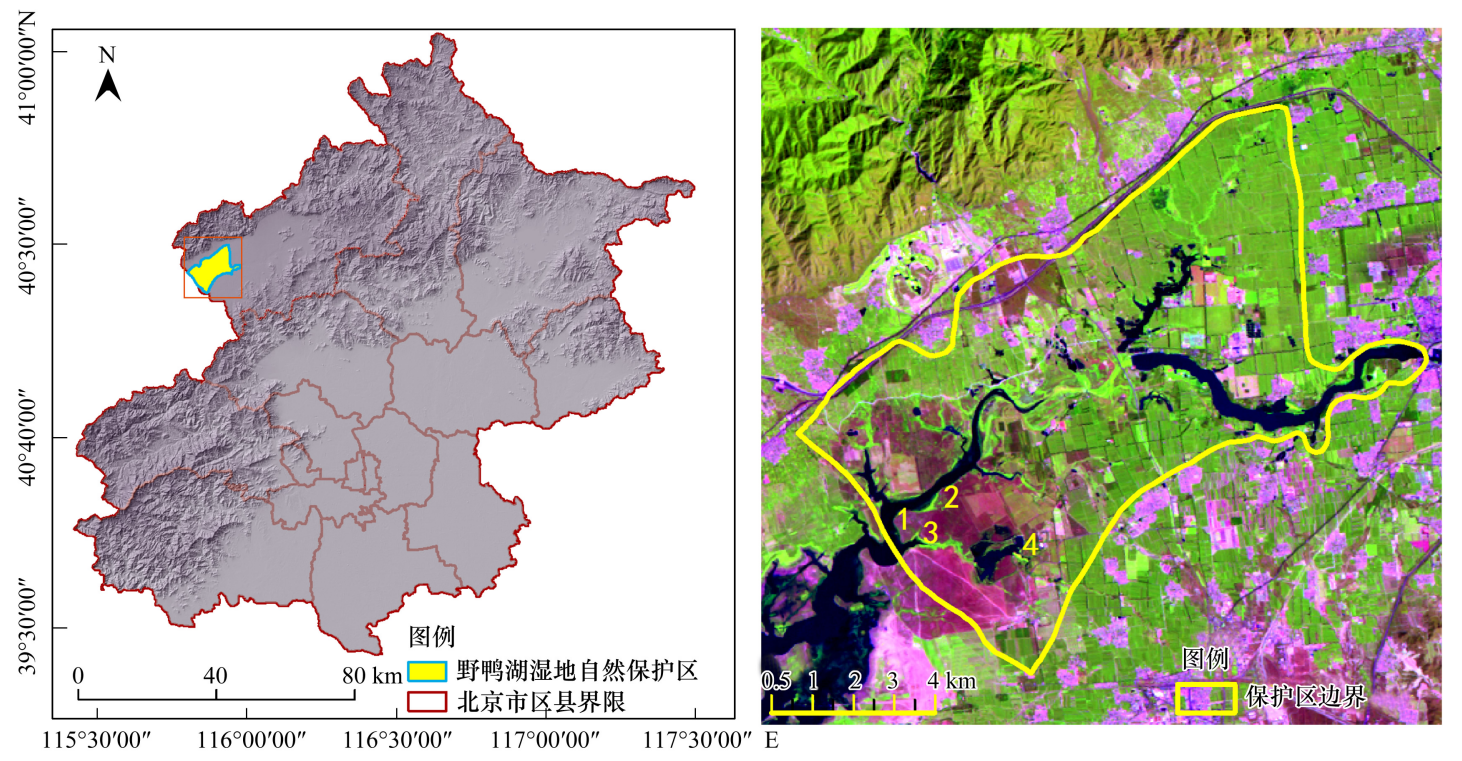

图 1 研究区地理位置图

Fig.1 Location map of study area

\section{2 采样区域与植被类型的选择}

湿地环境较为复杂, 有的区域因为地形地貌的 限制难以进人, 同时研究区内湿地植被类型众多, 因 此在采样过程中无法覆盖全部区域以及植被类型， 所以在进行野外光谱数据采集之前, 首先要对采样 区域及植被类型进行选择和确定。本次研究选定的 采样区域如图 1 所示,图 1 中 $1 、 2 、 3$ 三个区域内沟 岔众多、滩涂面积较大,湿地植被类型丰富,具有一
定的典型性和代表性; 区域 4 由人工建造的围堤所 包围, 围堤内湿地植被生长状况良好, 面积较大, 也 具有一定的典型性与代表性。上述区域内路面情况 较好,方便到达, 地势平坦, 水深较浅, 利于开展光谱 数据以及植被样本的采集工作。采样区域选定后, 结合研究目的和采样区域内湿地植物的类型、长势、 数量以及分布特点, 选择样区内的典型湿地植被进 行采样, 选定的典型植物有芦苇、香蒲、三叶鬼针草、 
刺儿菜、小叶杨。

\section{3 光谱数据的获取和预处理}

野外植物叶片光谱的测定采用采用美国 ASD-

Field Spec 3 光谱仪加载的手持叶夹式叶片光谱探测 器进行, 这种探测器内置石英卤化灯, 光源稳定; 测 量时叶片置于叶片夹的叶室中, 然后夹紧叶片, 保证 叶片水平且被探测面积相同, 以消除背景反射、叶片 表面弯曲造成的光谱波动及叶片内部变异造成的影 响,每组数据采集前均进行标准白板校正。光谱采 样的波长范围为 $350-2500 \mathrm{~nm}$, 其中 $350-1000 \mathrm{~nm}$ 和 $1000-2500 \mathrm{~nm}$ 的光谱采样间隔分别为 $1.4 \mathrm{~nm}$ 和 $2 \mathrm{~nm}$, 光谱分辨率分别为 $3 \mathrm{~nm}$ 和 $10 \mathrm{~nm}$, 数据采样间 隔为 $1 \mathrm{~nm}$, 光纤前视场角为 $25^{\circ}$ 。对测定的植物叶片 进行标识, 并注明编号。

光谱数据由于受到仪器以及目标地物本身光谱 特性等多种因素的影响, 获取的光谱数据中不仅包 含地物光谱信息,而且还包含噪声信息, 通过光谱数 据的预处理可以消除噪声并且突出地物光谱的某些 细微差别。光谱数据的预处理主要包括光谱平滑去 噪和光谱曲线均值处理两个部分。

(1) 光谱平滑去噪 由于光谱仪波段间对能量
响应上的差异, 使得光谱曲线总存在一些噪声, 为了 得到平稳与概略的变化, 需要平滑波形, 以去除包含 在信号内的少量噪声。本次研究中选用前后 9 个波 段的平均值作为滤波后的光谱值, 其函数式为:

$R_{i}^{\prime}=\frac{\left(R_{i-4}+R_{i-3}+R_{i-2}+R_{i-1}+R_{i}+R_{i+1}+R_{i+2}+R_{i+3}+R_{i+4}\right)}{9}$

(2) 光谱曲线均值处理 由于每个样点每次采 集 50 条光谱曲线,所以对每 10 条曲线进行一次均 值运算, 均值处理后的 5 条光谱曲线作为每个样点 的最终光谱曲线, 图 2 为本研究中获取的典型湿地 植物叶片光谱曲线。 5 种植物类型叶片光谱曲线的 主要差异主要存在于叶绿素强吸收的蓝、红光区域 和水吸收的中红外区域。在 350-700nm 区间主要 由叶片的各种色素控制叶片的反射率, 其中芦苇和 小叶杨的反射率最高; 在 700-1250nm 区间由细胞 结构控制的叶片反射率, 香蒲的反射率最高, 其次是 小叶杨; 在 1250-2300nm 区间由叶片含水量控制的 叶片反射率, 其中芦苇的反射率最高, 小叶杨次之; 总的来说芦苇、香蒲和小叶杨的光谱反射率较高而 刺儿菜的反射率最低。

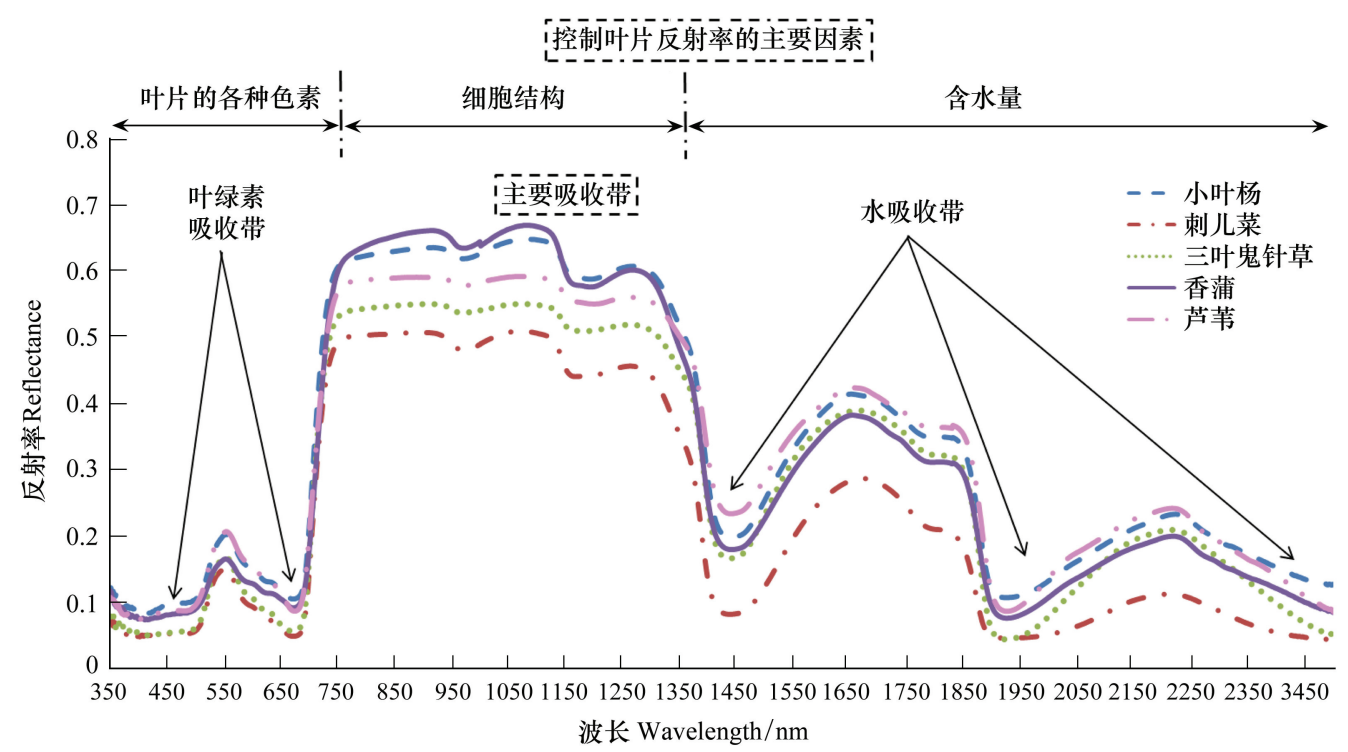

图 2 典型湿地植物叶片光谱曲线

Fig.2 Spectral curves of typical wetland plant vanes

\section{4 叶绿素含量的测定及分析}

叶绿素含量测量采用测量光谱的对应叶片中间 部位的叶脉两侧, 剪取 $0.30 \mathrm{~g}$ 叶片呈细丝状于试管 中, 以百分之一精度电子天平称量, 加人试剂 (丙
酮: 无水乙醇 $=1: 1$ ) $25 \mathrm{~mL}$, 闭光提取 $24 \mathrm{~h}$, 用 DR 2008 Spectrophotomete 分光光度计测 646 和 $663 \mathrm{~nm}$ 处的光 密度。计算叶绿素浓度:

叶绿素 $\mathrm{a}$ 浓度 $=13.95 \times \mathrm{A} 663-6.88 \times \mathrm{A} 646$ 
叶绿素 $\mathrm{b}$ 浓度 $=24.96 \times \mathrm{A} 646-7.32 \times \mathrm{A} 663$

叶绿素总浓度 $=\mathrm{Ca}+\mathrm{b}=\mathrm{Ca}+\mathrm{Cb}$

叶绿素含量 $=$ 叶绿素总浓度 $\times$ 浸提液体积 $\times$

稀释倍数/样品 (鲜)干质量

式中,A663、A646 分别代表在 663,646 波段处的光 密度值, 浓度单位 $\mathrm{mg} / \mathrm{L}$, 含量单位 $\mathrm{mg} / \mathrm{g}$ 。

水中的香蒲、岸边的芦苇、近岸的三叶鬼针草、 远岸的刺儿菜以及小叶杨在内的 5 种野鸭湖湿地常 见植物类型及对应的光谱曲线参见图 3。从图中可 以发现叶绿素含量最高的是芦苇, 其对应的光谱曲
线中在绿峰位置的光谱反射率也是最高的; 而叶绿 素含量较低的三叶鬼针草相对应的光谱曲线反射率 也最低。其中, 叶绿素含量相对含量较高的是芦苇、 香蒲和三叶鬼针草, 是野鸭湖湿地的典型湿地植物; 而叶绿素含量较低的小叶杨是中生植物 ${ }^{[23]}$ 。叶绿 素含量可以表征植物的营养状况, 同时也是植物受 胁迫及外界环境因子干扰状态下的指示器, 可以反 映植物的生产能力的高低。本研究结果表明在野鸭 湖湿地保护区中,其典型的湿生植物的叶绿素含量 相对较高,具有较高的生产力。
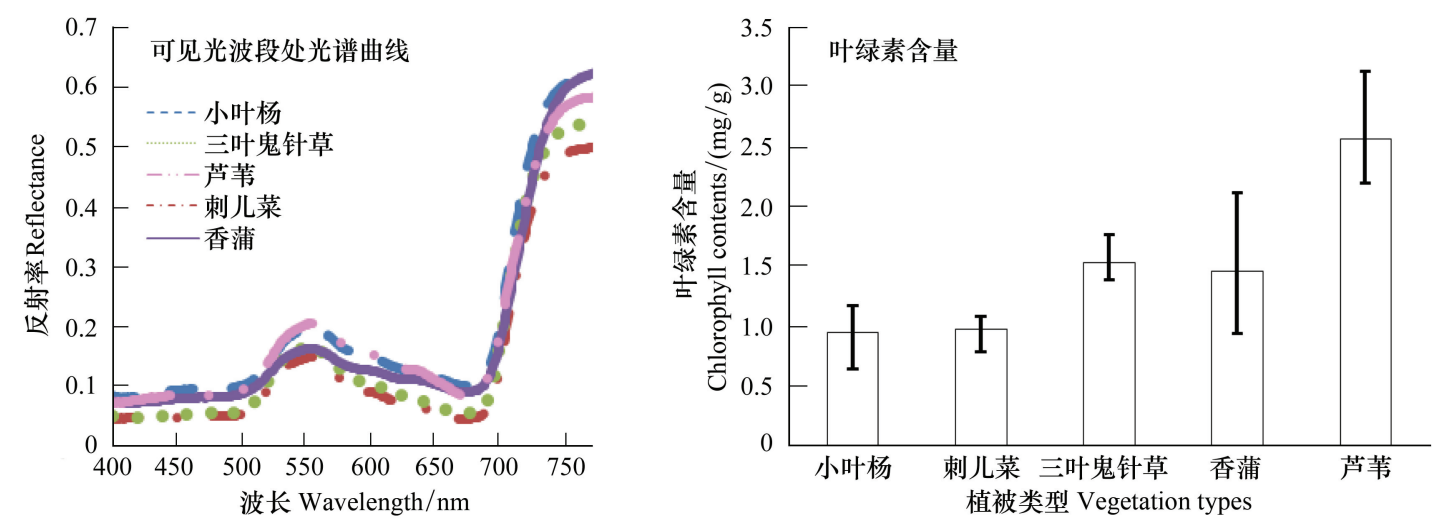

图 3 野鸭湖湿地典型植物类型叶绿素含量及可见光波段处光谱曲线

Fig.3 chlorophyll content of typical plants in Wild Duck Lake wetlands and the spectral curve at visible band

\section{5 数据分析方法}

以实测高光谱数据和叶绿素含量数据为基础, 首先选择植被生理生化参数反演模型常用的三边参 数,构建叶绿素高光谱反演模型。对选取的典型湿
地植物叶片的三边参数 (表 1) 与叶绿素含量进行相 关分析, 选择与叶绿素含量显著相关的光谱参数, 并 通过逐步回归的分析方法确定监测典型湿地植被的 最佳光谱参数,建立估算模型。

表 1 三边参数的定义

Table 1 Definition of trilateral parameters

\begin{tabular}{|c|c|c|}
\hline $\begin{array}{l}\text { 类型 } \\
\text { Type }\end{array}$ & $\begin{array}{l}\text { “三边”参数 } \\
\text { Trilateral parameters }\end{array}$ & $\begin{array}{l}\text { 定义 } \\
\text { Definition }\end{array}$ \\
\hline 基于光谱位置的变量 & Dr & 红边 $(680-760 \mathrm{~nm})$ 内最大一阶微分值 \\
\hline Characteristic variable based & WP_r & Dr 对应的波长即红边位置 \\
\hline \multirow[t]{4}{*}{ on spectral area } & $\mathrm{Db}$ & 蓝边 $(490-530 \mathrm{~nm})$ 内最大一阶微分值 \\
\hline & WP_b & Db 对应的波长即蓝边位置 \\
\hline & Dy & 黄边 $(560-640 \mathrm{~nm})$ 内最大一阶微分值 \\
\hline & WP_y & Dy 对应的波长即黄边位置 \\
\hline 基于光谱面积的变量 & SDr & 红边内一阶微分的总和 \\
\hline Characteristic variable based & $\mathrm{SDb}$ & 蓝边内一阶微分的总和 \\
\hline on spectral area & SDy & 黄边内一阶微分的总和 \\
\hline 基于植被指数形式的变量 & $\mathrm{SDr} / \mathrm{SDb}$ & 红边内一阶微分总和与蓝边内一阶微分总和的比值 \\
\hline Characteristic variable based & $\mathrm{SDr} / \mathrm{SDy}$ & 红边内一阶微分总和与黄边内一阶微分总和的比值 \\
\hline \multirow[t]{2}{*}{ on vegetation index } & $(\mathrm{SDr}-\mathrm{SDb}) /(\mathrm{SDr}+\mathrm{SDb})$ & 红边内一阶微分总和与蓝边内一阶微分总和的归一化值 \\
\hline & $(\mathrm{SDr}-\mathrm{SDy}) /(\mathrm{SDr}+\mathrm{SDy})$ & 红边内一阶微分总和与黄边内一阶微分总和的归一化值 \\
\hline
\end{tabular}


其次,为了使植被反射信息最大化、外部因素影 响最小化 ${ }^{[24]}$, 初步构建了比值植被指数和归一化差 值光谱指数。在光谱重采样的基础上 $(5 \mathrm{~nm})$, 本研 究初步构造了在 400-2350 nm 波段范围内由任意 两个波段反射率组合而成的所有比值 (6)、归一化 差值 (7), 使用 MATLAB 编程得到它们与植物叶绿 素含量的决定系数矩阵图, 分析它们与叶绿素含量 的关系:

$$
\begin{gathered}
S R=\frac{R_{n}}{R_{n+1}} \\
N D=\frac{R_{n+1}-R_{n}}{R_{n+1}+R_{n}}
\end{gathered}
$$

式中, $S R$ 为比值光谱指数, $N D$ 为归一化光谱指数, $R_{n}$ 为波段 $n$ 的反射率。

采用交叉检验 (Cross Validation,CV) 中的 K-fold Cross Validation 方法 (简称 K-CV) 对估算模型进行 测试和检验。 $\mathrm{K}-\mathrm{CV}$ 是将原始数据分成 $\mathrm{K}$ 组 (通常是 均分), 然后将每个子集数据分别做一次验证集, 其 余 $\mathrm{K}-1$ 组子集数据作为训练集,得到 $\mathrm{K}$ 个模型,最后 用这 $\mathrm{K}$ 个模型最终验证集的分类准确率平均数作为 此 K-CV 下分类器的性能指标。本次研究中所使用 的交叉检验方法为 $3 \mathrm{~K}-\mathrm{CV}$, 即将样本数据随机分成 3 组, 每组样点数量近似相等, 选取其中两组作为训练 样本建立估算模型,剩余一组进行验证,如此循环 3 次。估算模型的准确性和适用性采用通用的均方根
误差 (RMSE)、相对误差 ( RE) 和决定系数 $\left(R^{2}\right) 3$ 个 指标进行评定,并绘制预测值与实测值之间的 1:1 关 系图, 以直观的展示估算模型的拟合度和可靠性。 其中 $3 \mathrm{~K}-\mathrm{CV}$ 取 3 次验证的判定系数的平均值作为该 模型的精度。

\section{2 结果与分析}

2.1 基于 “三边” 参数的植物叶片叶绿素含量估算 模型

“三边” 参数能够较好的反映绿色植被的光谱特 征, 对叶绿素含量的变化较为敏感, 有学者指出, 红 边位置、红边平均反射率、红边位置附近平均反射 率、红边斜率、红边面积等反射光谱特征参数均与叶 绿素含量间存在指数函数关系。因此, “三边” 参数 可以作为叶绿素含量的诊断特征。利用与 “三边” 参 数有关的 13 个光谱特征变量与叶片的叶绿素含量 进行相关性分析。

从表 2 可以看出,基于光谱位置的变量中,在红 边位置 $\left(W P \_r\right)$ 与叶绿素含量呈现最大正相关 0.867 。基于光谱面积的变量中,在黄边面积 (SDy) 呈现了最大正相关 0.711 , 红边面积 $(\mathrm{SDr})$ 与叶绿素 含量不相关,相关系数为 0.054 。基于植被指数形式 的变量中,红边面积 (SDr) 与黄边面积 (SDy) 构建的 归一化植被指数的相关性最强, 相关系数达到 -0.581 , 呈极显著的负相关。

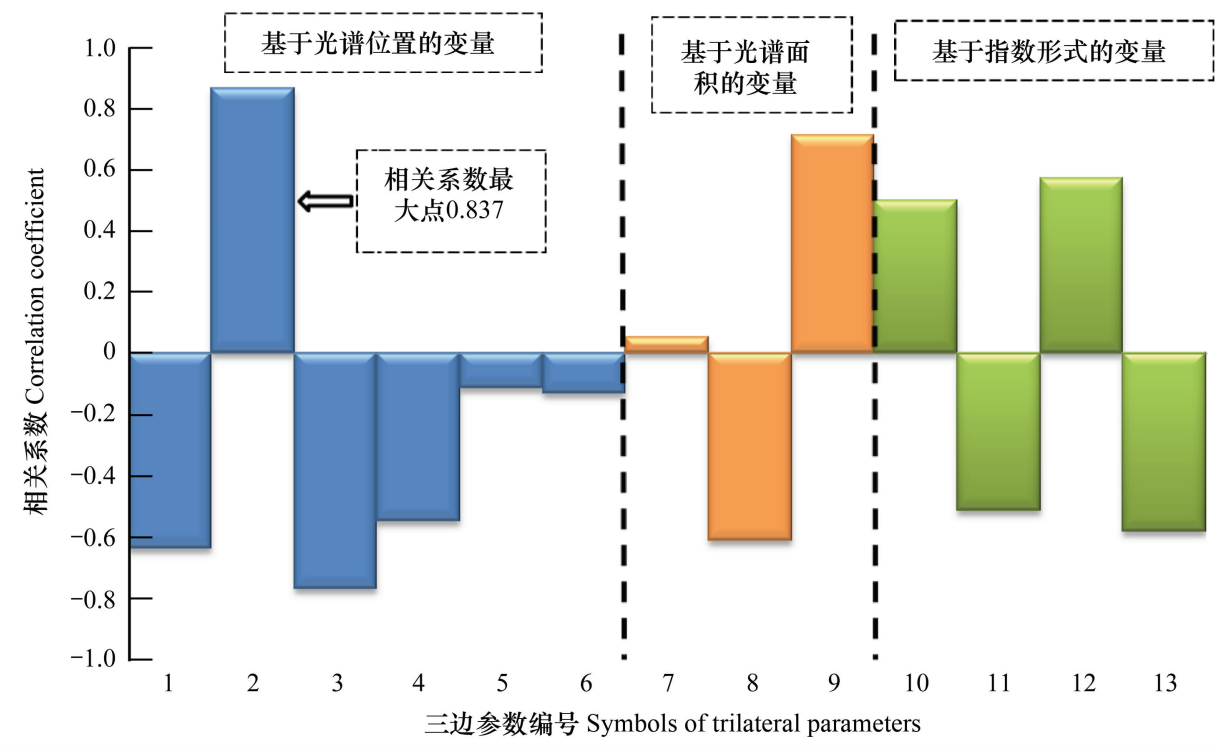

图 4 绿素含量与“三边”参数的相关系数

Fig.4 Correlation coefficient between chlorophyll content and trilateral parameters

1:Dr,2:WP_r, 3:Db ,4:WP_b ,5:Dy ,6:WP_y , 7:SDr, 8:SDb,9:SDy , 10:SDr/SDb , 11:SDr/SDy , 12:( SDr-SDb)/(SDr+SDb ) , 13: (SDr-SDy) / (SDr+SDy) 
表 2 植物叶绿素含量与“三边” 参数的相关系数

Table 2 Correlation coefficient between chlorophyll content and trilateral parameters

\begin{tabular}{llc}
\hline $\begin{array}{l}\text { 类型 } \\
\text { Type }\end{array}$ & $\begin{array}{l}\text { “三边”参数 } \\
\text { Trilateral } \\
\text { parameters }\end{array}$ & $\begin{array}{c}\text { 相关系数 } \\
\text { Correlation } \\
\text { coefficient }\end{array}$ \\
\hline 基于光谱位置的变量 & Dr & $-0.638^{* *}$ \\
Characteristic variable & WP_r & $0.867^{* *}$ \\
based on spectral area & Db & $-0.769^{* *}$ \\
& WP_b & $-0.547^{* *}$ \\
& Dy & -0.116 \\
基于光谱面积的变量 & WP_y & -0.133 \\
Characteristic variable & SDr & 0.054 \\
based on spectral area & SDy & $-0.614^{* *}$ \\
基于植被指数形式的变量 & SDr/SDb & $0.711^{* *}$ \\
Characteristic variable & SDr/SDy & $0.499^{* *}$ \\
based on vegetation index & $(\mathrm{SDr}-\mathrm{SDb}) /(\mathrm{SDr}+\mathrm{SDb})$ & $-0.515^{* *}$ \\
& $(\mathrm{SDr}-\mathrm{SDy}) /(\mathrm{SDr}+\mathrm{SDy})$ & $-0.573^{* *}$ \\
\hline
\end{tabular}

*表示在 0.05 水平上显著; $* *$ 表示在 0.01 水平上极显著, 样 本 $n=102$

植物叶片叶绿素含量与 “三边” 参数具有较好的 相关性,说明采用这些光谱特征变量对野鸭湖湿地 植被叶绿素含量进行估算具有较高的可行性,本文 中将采用交叉检验的方法 (3K-CV) 进行估算模型的 建立和验证。选取相关系数最大的光谱特征参数为 自变量 (红边位置 WP_r), 以叶绿素含量为因变量, 进行单变量线性回归分析, 建立叶绿素含量估算 模型。

表 3 叶绿素含量与最优“三边” 参数回归模型

Table 3 Regression model between chlorophyll content and best trilateral parameters

\begin{tabular}{ccc}
\hline $\begin{array}{c}\text { 光谱特征参数 }(x) \\
\text { Spectral } \\
\text { parameters }(x)\end{array}$ & $\begin{array}{c}\text { 回归方程 } \\
\text { Regression } \\
\text { equation }\end{array}$ & $R^{2}$ \\
\hline 红边位置(WP_r $)$ & $y=0.113 x-78.74$ & 0.751 \\
\hline 样本 $n=68$ & &
\end{tabular}

建立估算模型后, 为了检验估算模型的可靠性 和适用性,采用通用的均方根误差(RMSE)、相对误 差 $(\mathrm{RE})$ 和决定系数 $\left(R^{2}\right) 3$ 个指标对估算模型进行 评定 (表 4), 并绘制预测值与实测值之间的 1:1 关系 图(图 5 ), 以直观的展示估算模型的拟合度和可 靠性。

检验结果表明,基于红边位置( WP_r) 光谱特征 参数建立的叶绿素含量估算模型, 取得了较好的测

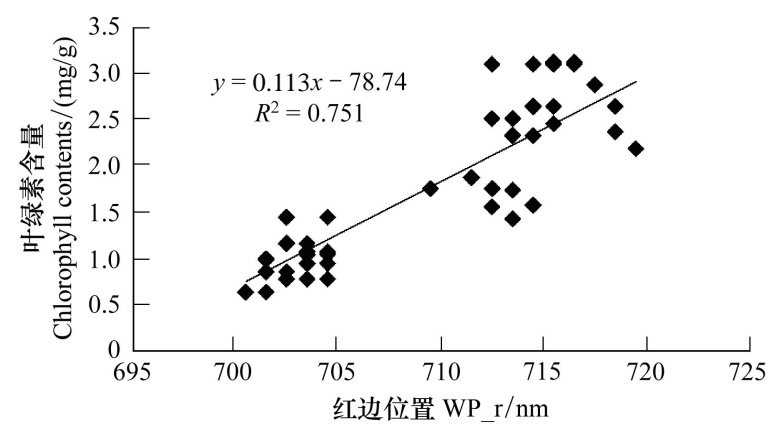

图 5 叶绿素含量与最优“三边”参数回归模型

Fig.5 Regression model between chlorophyll content and best trilateral parameters

试效果, 检验拟合方程的决定系数 $\left(R^{2}\right)$ 达到了 0.8 以上, 均方根误差 (RMSE) 和相对误差 (RE) 均较小, 具有较高的拟合精度和较小的相对误差。植被叶绿 素含量估算模型具有最大的决定系数 $\left(R^{2}=0.802\right)$ 、 最小的均方根误差 $(\mathrm{RMSE}=0.27)$ 和最小的相对误 差 $(\mathrm{RE}=6.1 \%)$ ，预测精度达到了 $93.9 \%$ 。

表 4 叶绿素含量实测值 $(x)$ 与预测值 $(y)$ 拟合评定指标

Table 4 The fit evaluation indicators of the measured values $(x)$ and predicted values $(y)$

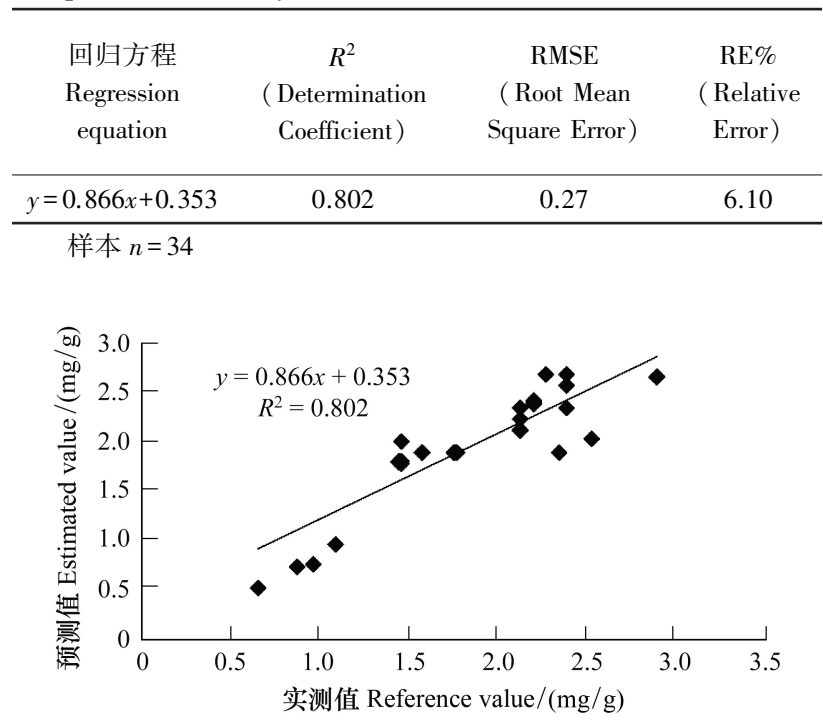

图 6 叶绿素含量实测值和预测值比较 ( “三边” 参数)

Fig. 6 Comparison between the measured value and the predicted value (trilateral parameters)

2.2 基于构建光谱指数的湿地植被叶绿素含量估 算模型

计算光谱反射率组成的比值和归一化光谱指数 与叶绿素含量的决定系数, 结果参见图 7 , 图中红色 代表相关系数最高, 蓝色代表相关系数最低。结果 显示归一化组合和比值组合之间具有一定的相似 
性,相关性达到 $P<0.01$ 显著水平, 总体相关性比较 高, 归一化指数优于比值指数, 相关系数高的比值组 合其归一化组合表现较好。较好的组合均为 550$700 \mathrm{~nm}$ 与 700-1400nm 以及 $550-700 \mathrm{~nm}$ 与 $1600-$ $1900 \mathrm{~nm}$ 。与叶绿素含量相关性最好的指数分别是

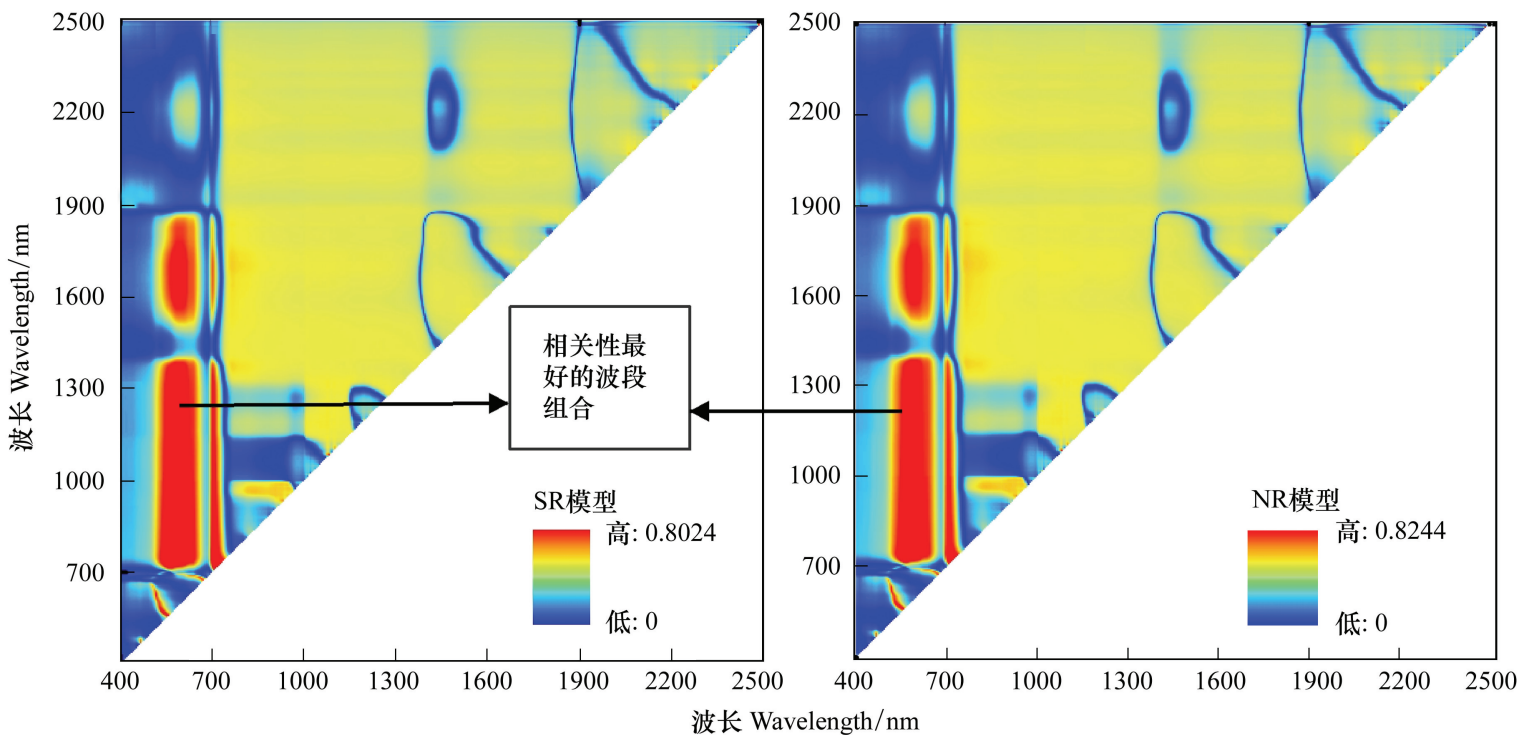

图 7 光谱指数与叶绿素含量的决定系数图

Fig.7 The coefficient of determination between the spectral index and chlorophyll content

表 5 叶绿素含量与最优光谱指数回归模型

Table 5 Regression model between chlorophyll content and best spectral index

\begin{tabular}{ccc}
\hline $\begin{array}{c}\text { 光谱特征参数 }(x) \\
\begin{array}{c}\text { Spectral } \\
\text { parameters }(x)\end{array}\end{array}$ & $\begin{array}{c}\text { 回归方程 } \\
\text { Regression } \\
\text { equation }\end{array}$ & $R^{2}$ \\
\hline $\mathrm{ND}(565,735)$ & $y=20.89 x-18.45$ & 0.824 \\
\hline
\end{tabular}

样本 $n=20$

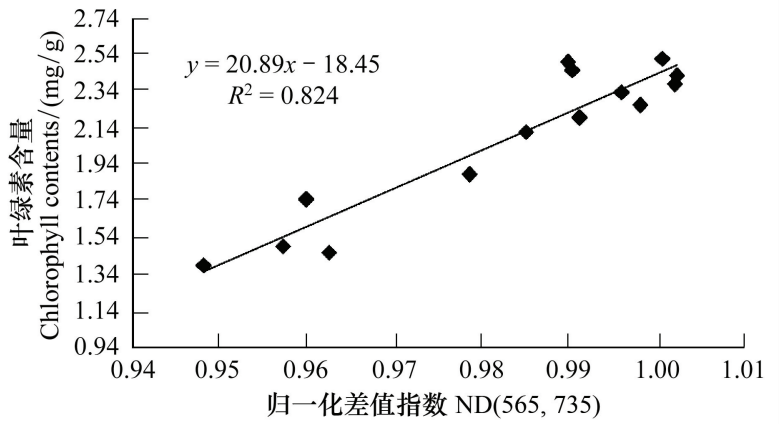

图 8 叶绿素含量与最优光谱指数回归模型

Fig.8 Regression model between chlorophyll content and best spectral index

检验结果表明, $\operatorname{ND}(565,735)$ 光谱指数的叶绿 素含量估算模型, 检验拟合方程的决定系数 $\left(R^{2}\right)$ 达 到了 0.8 以上, 均方根误差 (RMSE) 和相对误差
$\mathrm{SR}(565 \mathrm{~nm}, 740 \mathrm{~nm})$ 和 $\mathrm{ND}(565 \mathrm{~nm}, 735 \mathrm{~nm})$ 。利用最 优的光谱指数 $\operatorname{ND}(565,735)$ 与叶绿素含量建立线性 回归模型,并对模型进行交叉验证,交叉验证的决定 系数和均方根误差如表 6 所示。 


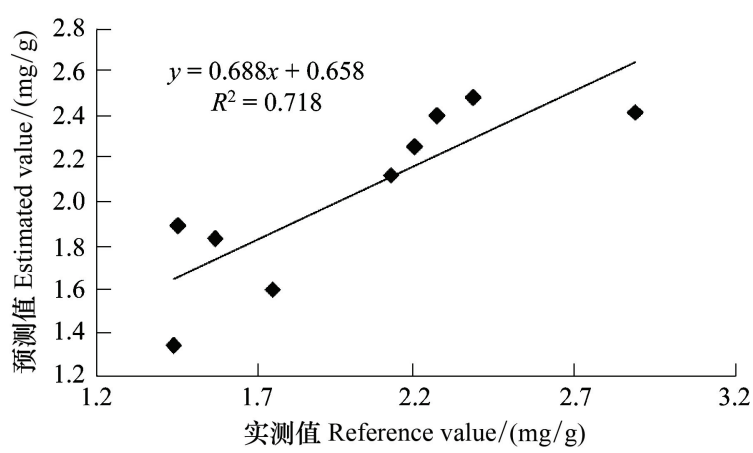

图 9 叶绿素含量实测值和预测值比较 $(\mathrm{ND}(565,735))$

Fig. 9 Comparison between the measured value and the predicted value $(\mathrm{ND}(565,735))$

绿素含量估算模型,并采用交叉检验中的 $3 \mathrm{~K}-\mathrm{CV}$ 方 法对估算模型进行了测试和检验。结论如下:

(1) 植物叶片叶绿素含量选取的光谱特征参数 整体上相关性较强, 与 “三边” 参数大多都呈极显著 相关, 相关系数最大达到 0.867 ; SR 和 ND 光谱指数 与叶绿素含量的决定系数, 总体相关性比较高, 与叶 绿素含量相关性最好的指数分别是 SR $(565 \mathrm{~nm}$, $740 \mathrm{~nm})$ 和 $\mathrm{ND}(565 \mathrm{~nm}, 735 \mathrm{~nm})$, 相关系数分别达到 $0.8024 、 0.8244$ 。说明采用这些光谱特征变量对植被 叶绿素含量进行估算具有较高的可行性。

(2) 通过选取相关性最佳的光谱特征参数, 分别 基于 “三边” 参数和光谱植被指数构建了植物叶片叶 绿素估算模型。其中,基于红边位置(WP_r) 光谱特 征参数和 $\mathrm{ND}(565 \mathrm{~nm}, 735 \mathrm{~nm})$ 光谱指数建立的叶绿 素含量估算模型, 取得了较好的测试效果, 检验拟合 方程的决定系数 $\left(R^{2}\right)$ 达到了 0.8 以上,估算模型分 别为 $y=0.113 x-78.74, y=5.5762 x+4.4828$ 。

(3) 本次研究的估算模型采用交叉检验中的 $3 \mathrm{~K}-\mathrm{CV}$ 方法进行测试和检验, 3K-CV 方法可以充分 利用样本数据, 有效的避免过学习以及欠学习状态 的发生, 得到的结果也更具有说服力。以上两方面 较大的增强了模型的可靠性和普适性。通过测试和 检验, 植物叶绿素含量估算模型均取得了较为理想 的预测精度, 预测精度的分别为 $93.9 \%$ 及 $90.07 \%$, 为 遥感技术在野鸭湖湿地植物含水量诊断中的实际应 用提供理论依据和技术支持。

(4) 本次研究采用高光谱遥感分析估算湿地植 物叶绿素含量,该方法属于一种无损测试技术, 是在 不破坏植物体本身组织结构的前提下, 利用高光谱 辐射仪测得叶片叶绿素光谱反射特征变化。研究中
还发现, “三边” 参数与叶绿素含量拟和方程的拟合 度相对高于高光谱植被指数与之建立的方程的拟合 度, 说明 “三边” 参数的光谱特征参数更适合叶绿素 含量的判别。

(5) 本文基于光谱指数的叶绿素含量估算, 只 是分析了 400-2500 nm 范围内任意单波段或者两 波段组合而成的比值 $(S D)$ 和归一化 (ND) 光谱指数 与叶绿素含量的关系,并且所得模型精度低于基于 三边参数构建的叶绿素含量估算模型。因此, 在下 一步研究中,需要进一步分析多波段 (三波段或者更 多)组合而成的光谱指数与叶绿素含量的相关性,并 且采用多元回归分析拟合方法, 构建相关性更高的 光谱植被指数,进一步提高估算模型的可靠性和准 确性; 另一方面, 利用统计学方法得到的经验模型, 虽然具有一定的可靠性和普适性，但是在时间域和 空间域内仍然具有较大的局限性,需要利用理论基 础更为严密的辐射传输模型 (Prospect 模型), 进行 叶绿素含量的估算; 最后, 本文只应用实测高光谱数 据进行分析,无法体现宏观范围上的湿地植物叶绿 素含量分布,在下一步研究中应结合星载或机载高 光谱影像, 达到图谱合一,充分发挥高光谱遥感技术 的特点和优势。

\section{References :}

[ 1 ] Marion L, Paillison J M. A mass balance assessment of the contribution of floating-leaved macrophytes in nutrient stocks in an eutrophic macrophyte-dominated lake. Aquatic Botany, 2003, 75 (3) : 249-260.

[2] Pan Y Z, Shi W Q, Zhu W Q, Gu X H, Fan Y D, Li J. Measurement of ecological capital of Chinese terrestrial ecosystem based on remote sensing. Science in China (Series D), 2005, 48 (6) : 786-796

[ 3 ] Minoha Co. Ltd. Chlorophyll SPAD-02 Instruction Manual. Tokyo : Radiometric Instruments Operations, 1989.

[ 4 ] Vane G, Goetz A F H. Terrestrial imaging spectrometry : Curren status, future trends. Remote Sensing of Environment, 1993, 44 $(2 / 3): 109-127$

[ 5 ] Fassnacht K S, Gower S T, Mackenzie M D, Nordheim E V, Lillesand T M. Estimating the leaf area index of north central Wisconsin forests using the landsate thematic mapper. Remote Sensing of Environment, 1997, 61(2) : 229-245.

[ 6 ] Wang J H, Zhao C J, Huang W J. The Basis and Application of Quantitative Remote Sensing in Agriculture. Beijing: Science and Technology Press, 2008: 4-5.

[ 7 ] Guizar-Sicairos M, Thurman S T, Fienup J R. Efficient subpixel image registration algorithms. Optics Letters, 2008, $33(2)$ : 156- 158. 
[ 8 ] Soummer R, Pueyo L, Sivaramakrishnan A, Vanderbei R J. Fast computation of lyot-style coronagraph propagation. Optics Express, 2007, 15(24) : 15935-15951.

[ 9 ] Fang H, Song H Y, Cao F, He Y, Qiu Z J. Study on the relationship between spectral properties of oilseed rape leaves and their chlorophyll content. Spectroscopy and Spectral Analysis, 2007, 29(9): 1731-1734.

[10] Steven M D, Clark J A, Demetriades-Shah T H, Danson F M, Clark J A. High-spectral resolution indices for crop stress. Application of Remote Sensing in Agriculture. Sevenoaks: Butterworths, 1990: 209-227.

[11] Horler D N H, Dockray M, Barber J. The red edge of plant leaf reflectance. International Journal of Remote Sensing, 1983, 4 (2) : 273-278.

[12] Curran P J, Dungan J L, Gholz H L. Exploring the relationship between reflectance red edge and chlorophyll content in Slash pine. Tree Physiology, 1990, 7(1/4) : 33-48.

[13] Du H Q, Ge H L, Fan W Y, Jin W, Zhou Y F, Li J. Study on relationships between total chlorophyll with hyperspectral features for leaves of Pinus massoniana forest. Spectroscopy and Spectral Analysis, 2009, 29(11) : 3033-3037

[14] Tang Y L, Huang J F, Wang X Z, Cai S H. Study on hyper spectral characteristics of corn leaves and their correlation to chrolophyll and carotenoid. Journal of Maize Sciences, 2008, 16 (2) : 71-76

[15] Wu D, Huang L X, He Y, Pan J Z, Zhang B. Visible-near infrared reflection spectroscopy for crop-weed discrimination. Acta Optica Sinica, 2008, 28(8) : 1618-1622.

[16] Song K S, Zhang B, Wang Z M, Liu H J, Duan H T. Inverse model for estimating soybean chlorophyll concentration using insitu collected canopy hyperspectral data. Transactions of the Chinese Society of Agricultural Engineering, 2006, 22 ( 8 ): 16-21.

[17] Yi Q X, Huang J F, Wang X Z, Qian L. Hyperspectral remote sensing estimation models for chlorophyll concentration in corn. Bulletin of Science and Technology, 2007, 23(1) : 83-87.

[18] Zhao X, Liu S H, Wang P J, Tian Z K. A method for inverting chlorophyll content of wheat using hyperspectral. Geography and Geo-Information Science, 2004, 20(3) : 36-39.

[19] Tang Y L, Wang R C, Huang J F, Kong W S, Cheng Q. Hyperspectral data and their relationships correlative to the pigment contents for rice under different nitrogen support level. Journal of Remote Sensing, 2004, 8(2): 185-192.

[20] Liu W D, Xiang Y Q, Zheng L F, Tong Q X, Wu C S. Relationships between rice LAI, CH. D and hyperspectra data. Journal of Remote Sensing, 2000, 21(4) : 279-283.

[21] Huang C Y, Wang D W, Yan J, Zhang Y X, Cao L F, Cheng C. Monitoring of cotton canopy chlorophyll density and leaf nitrogen accumulation status by using hyperspectral data. Acta Agronomica Sinica, 2007, 33(6): 931-936.
[22] Chen W, Hu D, Fu B Q. Beijing Wetland Biodiversity Research. Beijing: Science and Technology Press, 2007: 47-54.

[23] Gong Z N, Gong H L, Hu D. Wetland Plants in Wild Duck Lake in Beijing. Beijing: China Environmental Science Press, 2012: 116- 117.

[24] Yang J, Tian Y C, Yao X, Cao W X, Zhang Y S, Zhu Y. Hyperspectral estimation model for chlorophyll concentrations in top leaves of rice. Acta Ecologica Sinica, 2009, 29 ( 12 ): $6561-6571$.

\section{参考文献:}

［2］潘耀忠, 史培军, 朱文泉, 顾晓鹤, 范一大, 李京. 中国陆地 生态系统生态资产遥感定量测量. 中国科学 D 辑, 2004, 34 (4) : 375-384.

[6]王纪华, 赵春江, 黄文江. 农业定量遥感基础与应用. 北京: 科学出版社, 2008: 4-5.

[9] 方慧, 宋海燕, 曹芳, 何勇, 表正军. 油菜叶片的光谱特征与 叶绿素含量之间的关系研究. 光谱学与光谱分析, 2007, 27 (9): 1731-1734.

［13］杜华强, 葛宏立, 范文义, 金伟, 周宇峰, 李进. 马尾松针叶 光谱特征与其叶绿素含量间关系研究. 光谱学与光谱分析, 2009, 29(11): 3033-3037.

[14] 唐延林, 黄敬峰, 王秀珍, 蔡绍洪. 玉米叶片高光谱特征及与 叶绿素、类胡夢卜素相关性的研究. 玉米科学, 2008, 16(2): 71-76.

[15] 吴迪, 黄凌霞, 何勇, 潘家志, 张埙. 作物和杂草叶片的可见近红外反射光谱特性. 光学学报, 2008, 28(8)：1618-1622.

[16] 宋开山, 张柏, 王宗明, 刘焕军, 段洪涛. 大豆叶绿素含量高 光谱反演模型研究. 农业工程学报, 2006, 22(8) : 16-21.

[17] 易秋香, 黄敬峰, 王秀珍, 钱翌. 玉米叶绿素高光谱遥感估算 模型研究. 科技通报, 2007, 23(1)：83-87, 105-105.

[18] 赵祥, 刘素红, 王培娟, 王锦地, 田振坤. 基于高光谱数据的 小麦叶绿素含量反演. 地理与地理信息科学, 2004, 20(3): 36-39.

[19］唐延林, 王人潮, 黄敬峰, 孔维姝, 程乾. 不同供氮水平下水 稻高光谱及其红边特征研究. 遥感学报, 2004, 8 (2): 185- 192.

[20］刘伟东, 项月琴, 郑兰芬, 童庆禧, 吴长山. 高光谱数据与水 稻叶面积指数及叶绿素密度的相关分析. 遥感学报, 2000, 21 (4) : 279-283.

[21] 黄春燕, 王登伟, 间洁, 张显星, 曹连莆, 程诚. 棉花叶绿素 密度和叶片氮积累量的高光谱监测研究. 作物学报, 2007, 33 (6) : 931-936.

[22] 陈卫, 胡东, 付必谦. 北京湿地生物多样性研究. 北京: 科学 出版社, 2007: 47-54.

[23] 宫兆宁, 宫辉力, 胡东. 北京野鸭湖湿地植物. 北京: 中国环 境科学出版社, 2012: 116-117.

[24］杨杰, 田永超, 姚霞, 曹卫星, 张玉森, 朱艳. 水稻上部叶片 叶绿素含量的高光谱估算模型. 生态学报, 2009, 29(12): 6561-6571. 